\title{
REVIEWS
}

\section{Prevention of Post-operative Delirium in the Elderly Using Pharmacological Agents}

\author{
Patrice Tremblay, MD, CCFP (COE) $)^{1}$, Susan Gold, MD, FRCPC ${ }^{2}$ \\ ${ }^{1}$ Department of Family Medicine, St. Mary's Hospital Center, Mc Gill University, Montreal, PQ; \\ ${ }^{2}$ Department of Geriatric Medicine, Jewish General Hospital, McGill University, Montreal, PQ
}

DOI:http://dx.doi.org/10.5770/cgj.19.226

\begin{abstract}
\section{Introduction}

Post-operative delirium (POD) is a serious surgical complication that can cause significant morbidity and mortality. It is associated with prolonged hospital stay, delayed admission to rehabilitation programs, persistent cognitive deficits, marked health-care costs, and more. The pathophysiology is multifactorial and not completely understood, which complicates the optimal management. Non-pharmacological measures have been the mainstay of treatment, but there has been an ongoing interest in the medical literature on the prevention of post-operative delirium using medications. The purpose of this review is to critically analyze the current evidence on pharmacological prevention of POD.
\end{abstract}

\section{Methods}

A literature review was conducted using PubMed and Embase databases, using the following search terms: delirium, antipsychotics, cholinesterase inhibitors, and statins.

\section{Results}

A total of 1,152 articles were screened and 25 articles were reviewed. Fourteen articles found a reduced incidence of post-operative delirium using pharmacological agents: eight with antipsychotics, two with statins, one with melatonin, one with dexamethasone, one with gabapentin, and one with diazepam. However, study designs, methodological issues, or authors' interpretations raise questions on these conclusions.

\section{Conclusions}

Further double-blinded randomized clinical trials should be conducted before administering pharmacological agents to reduce POD in a non-research setting.

Key words: delirium, post-operative, prevention, pharmacological agents

\section{INTRODUCTION}

Delirium is a neuropsychiatric disorder characterized by a sudden onset of altered and fluctuating level of consciousness, inattention, and disorganized thinking. ${ }^{(1)}$ The etiology is organic and sometimes modifiable. Surgery, especially high operative stress procedures such as major joint replacements or cardiovascular surgery, is a strong risk factor. The incidence of post-operative delirium (POD) is extremely variable; a review of 80 primary studies showed a range from $0 \%$ to $73.5 \%$ (average 36.8\%). ${ }^{(2)}$ The factors associated with POD may be divided into pre-operative, perioperative, and post-operative as demonstrated in Table 1. ${ }^{(3)}$

Delirium is harmful to the patient, and costly to the health-care system. It increases the risk of long-term cognitive impairment, loss of autonomy, prolonged hospital length of stay, and societal cost. ${ }^{(4,5)}$ Although it is documented that non-pharmacological measures and comprehensive geriatric assessments reduce the risk of post-operative delirium, the evidence for pharmacological agents seems less convincing. (6) Given the lack of human resources in hospitals, the idea that a medication could reduce the incidence of post-operative delirium is interesting and potentially time-saving. Different classes of medications which have been studied so far include antipsychotics, cholinesterase inhibitors, statins, melatonin, steroids, and anticonvulsants. The conclusions drawn are conflicting, thus we proceeded to a literature review.

\section{METHODS}

A literature review was conducted using the databases PubMed, and Embase. The literature published from January 1, 1999 to December 31, 2014 was searched using the following terms: delirium, post-operative delirium, post-operative cognitive dysfunction, surgical procedures, post-operative period, post-operative complications, antipsychotics, haloperidol, quetiapine, risperidone, olanzapine, cholinesterase inhibitors, donepezil, galantamine, rivastigmine, memantine, statins, hydroxymethylglutaryl coenzyme A reductase inhibitor, and melatonin. This time period was determined because the first relevant article was published in 1999. ${ }^{(7)}$ A total of

(C) 2016 Author(s). Published by the Canadian Geriatrics Society. This is an Open Access article distributed under the terms of the Creative Commons Attribution Non-Commercial No-Derivative license (http://creativecommons.org/licenses/by-nc-nd/2.5/ca/), which permits unrestricted non-commercial use and distribution, provided the original work is properly cited. 
1152 potentially relevant articles were identified. After an evaluation based on the titles and the abstracts, 1110 articles were excluded if the delirium was not in a post-operative period, if the preventative measures were solely non-pharmacological, if the pharmacological agent was used only to treat delirium and not to prevent it, and if the patients enrolled were children. Case reports were also excluded. Forty-two articles remained available for a full-text evaluation. Twenty articles were duplicates and were therefore removed from the primary studies. Finally, three articles were identified from the references of the other studies selected. The final number of articles reviewed in this study was 25 , as demonstrated in Figure 1.

TABLE 1. Major POD risk factors

\begin{tabular}{lcc}
\hline \multicolumn{1}{c}{ Pre-operative } & Peri-operative & Post-operative \\
\hline $\begin{array}{l}\text { Cognitive } \\
\text { impairment }\end{array}$ & $\begin{array}{c}\text { General } \\
\text { anesthesia }\end{array}$ & Narcotics \\
Older age & Brain hypoperfusion & Sleep deprivation \\
Polypharmacy & $\begin{array}{l}\text { Hemodynamic } \\
\text { instability }\end{array}$ & Pain \\
$\begin{array}{l}\text { Electrolytes and } \\
\text { glucose disturbances }\end{array}$ & $\begin{array}{l}\text { High stress } \\
\text { procedures }\end{array}$ & Infections \\
\hline
\end{tabular}

\section{RESULTS}

\section{Antipsychotics}

Antipsychotics are the most-studied medications for the prevention of POD. It has been speculated that an excess of dopamine contributes to the development of delirium. In delirious patients, several metabolic pathways under hypoxic conditions lead to an increased production of dopamine, as well as a reduced degradation of dopamine. ${ }^{(8)}$ Its cerebral accumulation results clinically in delirium. Therefore, administrating antipsychotics, which are dopamine D2 receptor antagonists, could theoretically prevent POD. Ten studies have been published in this regard that include three meta-analyses, $(9,10,11)$ four randomized controlled trials with haloperidol, $(7,12,13,14)$ two with risperidone, ${ }^{(15,16)}$ and one with olanzapine. ${ }^{(17)}$ The mean age range varied from 61 to 80 years old.

\section{Haloperidol}

Regarding haloperidol, two studies showed a reduction of post-operative delirium. ${ }^{(7,13)}$ Kaneko et al. ${ }^{(7)}$ published the first trial in 1999 using intravenous haloperidol at a dose of $5 \mathrm{mg}$ intravenously daily for five consecutive days post-operatively. Eighty patients scheduled for elective gastrointestinal surgery and admitted to the Intensive Care Unit at Tottori University Hospital in Japan, between 1995 and 1998, were initially enrolled. Seventy-eight patients completed the

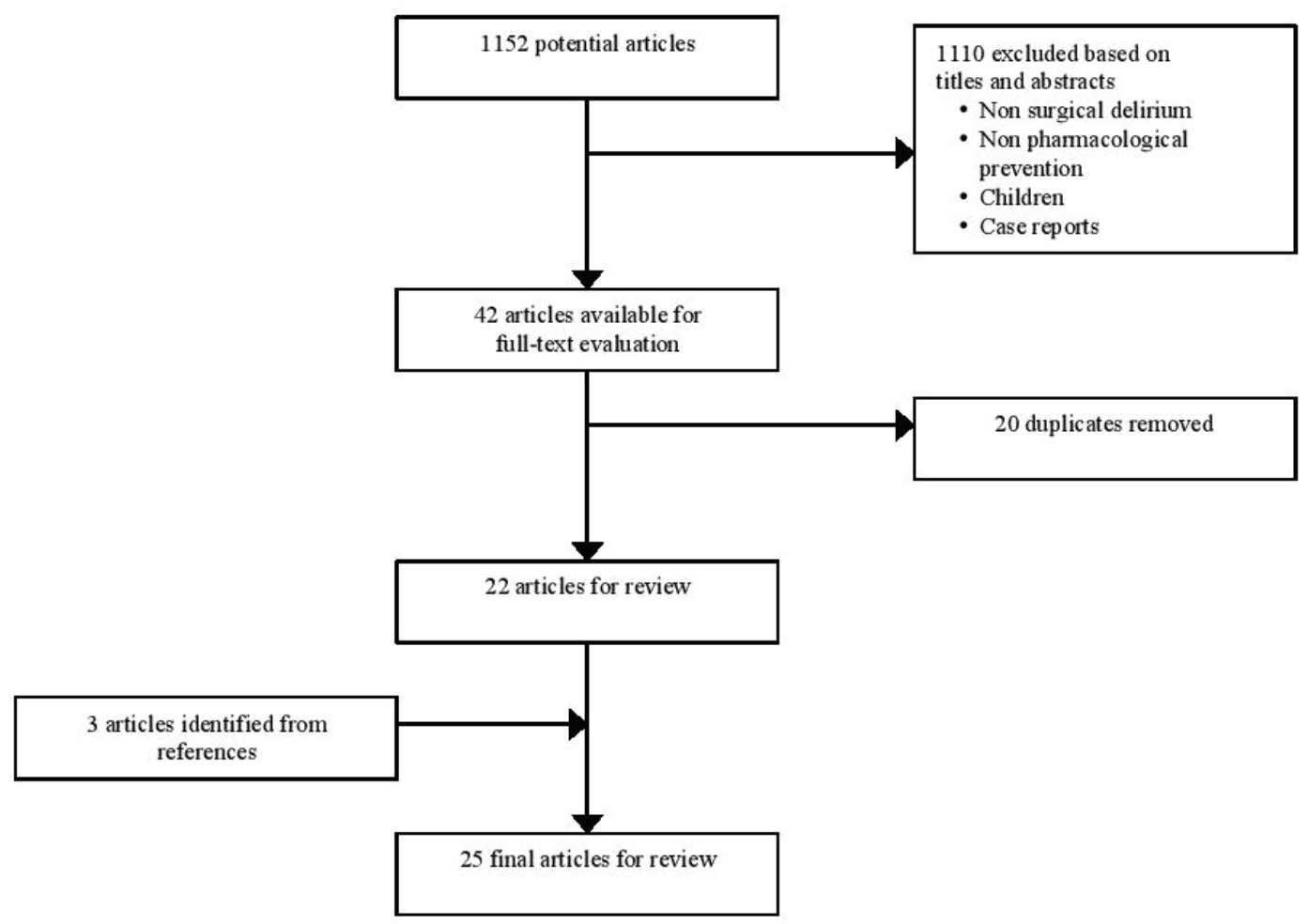

FIGURE 1. Articles screening process 
study (intervention group $n=38$, control group $n=40$ ). Two patients had to be excluded because they were admitted in the Intensive Care Unit (ICU) before the randomization. The control group received intravenous normal saline. The mean age was 72.4 years old in the haloperidol group and 73.1 years old in the placebo group. The patients underwent partial gastrectomy ( $n=20$ in haloperidol group, $n=23$ in saline group), total gastrectomy ( $n=8$ in haloperidol group, $n=6$ in saline group), or colectomy ( $n=10$ in haloperidol group, $n=11$ in saline group). The diagnosis of delirium was made based on the Diagnostic and Statistical Manual of Mental Disorders criteria, DSM III-R. Post-operative delirium developed in 17 patients; 4 in the study group and 13 in the placebo group. The post-operative delirium began two to four days after the surgery. The incidence of delirium was significantly reduced in the study group using haloperidol compared to placebo $(10.5 \%$ versus $32.5 \%, p<.05)$ with no major side effects found. Specifically, there were no extrapyramidal side effects. However, the study was non-blinded and the number of patients who completed the study was small $(n=78)$. Wang et al. ${ }^{(13)}$ completed a larger study with 457 patients 65 years or older undergoing non-cardiac surgery (intra-abdominal, intra-thoracic, spinal and extremital, superficial) with an ICU stay. The study was conducted from June 2009 to May 2010 in ICUs of two large tertiary teaching hospitals in Beijing, China. Exclusion criteria included patients undergoing neurosurgery, patients with parkinsonism, previous use of antipsychotics, profound dementia, use of cholinesterase inhibitor, and history of schizophrenia. The diagnosis of delirium was made using the confusion assessment method for the ICU. It was a double-blinded randomized controlled trial where the intervention group $(n=229)$ received a bolus of haloperidol $0.5 \mathrm{mg}$ followed by a drip infusion of $0.1 \mathrm{mg} /$ hour for 12 hours for a total of $1.7 \mathrm{mg}$ intravenously, compared to a placebo group $(n=228)$. The control group received normal saline. The mean age of both groups was 74 years old. A multicomponent approach to reduce risk factors of delirium was included in routine care, and non-pharmacologic strategies were applied first for patients who had developed delirium. The primary endpoint was the incidence of delirium during the first seven days after surgery. Within these seven days, 35 patients $(15.3 \%)$ in the haloperidol and 40 patients $(17.5 \%)$ in the placebo group developed post-operative delirium. There was a statistically significant decreased incidence of post-operative delirium in the haloperidol group compared to placebo (15.3\% versus $23.2 \%$, $p=.031)$. The intervention group also had a decreased ICU length of stay of 1.7 hour, which was statistically significant $(p=.024)$. However, the length of stay in hospital did not differ between the groups (11.0 days in both groups). Fukata et $_{\text {al. }}{ }^{(12)}$ enrolled 121 patients, 75 -years-old or older, who underwent elective abdominal surgery under general anesthesia or elective orthopedic surgery under general or spinal anesthesia, between January 2007 and December 2012 in Japan. It was a randomized, open-label prospective trial where the intervention group received haloperidol intravenously by drip infusion once daily $(2.5 \mathrm{mg} /$ day $)$ from post-operative days 1 to 3 . The non-intervention group did not receive preventive treatment. Delirium was assessed using the NEECHAM confusion scale, where a score below 20 (maximum score 30) after surgery was indicative of post-operative delirium. Patients who underwent emergency surgery, newly added or switched psychotropic medications within two weeks prior to surgery, or patients treated with haloperidol for delirium after surgery before the preventive administration, were excluded. In total, 59 patients were allocated in the intervention group ( 52 abdominal surgeries, 5 orthopedic surgeries, and 2 others) and 62 patients in the placebo group (55 abdominal surgeries, 4 orthopedic surgeries, and 3 others). Two patients used haloperidol on post-operative day 0 for therapeutic purposes in the control group, and had to leave the study. Therefore, 119 patients completed the study. The mean ages were 80.5 years in the intervention group and 80.2 years in the control group. The incidence of post-operative delirium in all patients was $37.8 \%$. The incidence was not significantly lower in the intervention group than in the control group (33.3\% in intervention group versus $42.4 \%$ in control group, $p=.309)$. Kalisvaart et al. ${ }^{(14)}$ published a randomized, placebo-controlled, double-blind, clinical trial of low-dose haloperidol prophylaxis for post-operative delirium in elderly hip-surgery patients. Men and women aged 70 and older admitted for acute or elective hip surgery were considered for inclusion between August 2000 and August 2002 in a teaching hospital in the Netherlands. Four hundred and thirty patients were randomized, and 395 patients completed the study (intervention $n=201$, placebo $n=194$ ). Patients were excluded if they had delirium at admission, no risk factors for post-operative delirium, use of cholinesterase inhibitors, parkinsonism, profound dementia, epilepsy, levodopa treatment, or prolonged QTc. Delirium was diagnosed using the criteria of Diagnostic and Statistical Manuel of Mental Disorder, Fourth Edition (DSM-IV) and Confusion Assessment Method (CAM) criteria. The intervention group received $1.5 \mathrm{mg}$ of haloperidol by mouth daily for three consecutive post-operative days. Experienced geriatric nurses and geriatricians provided geriatric consultation to all patients. The overall incidence of post-operative delirium was $15.8 \%$. There was no significant change in the incidence of delirium between the intervention and control group $(15.1 \%$ versus $16.5 \%$, respectively, relative risk $=0.91$, $95 \% \mathrm{CI}=0.59-1.44)$. However, the duration of delirium was less in the intervention group compared to placebo (5.4 days versus 11.8 days, $p<.001)$, as well as mean number of days in the hospital (mean difference 5.5 days, $p<.001$ ).

\section{Risperidone}

The two double-blinded randomized controlled trials of risperidone showed promising results. ${ }^{(15,16)}$ Prakanrattana and Prapaitrakool ${ }^{(16)}$ enrolled 126 patients aged over 40 years who 
had elective cardiac surgery under cardiopulmonary bypass (coronary artery bypass graft, valve, other). It was a randomized, double-blinded, placebo-controlled study. Patients who were to undergo emergency surgery, admitted to the intensive care unit, diagnosed with pre-operative delirium, or previous documented history of psychiatric problems were excluded. No patient was unable to participate in the study. The population was the youngest among the antipsychotics studies, with a mean age of 61 years old for both experimental and control groups. The patients were given either $1 \mathrm{mg}$ of risperidone sublingual $(n=63)$ or placebo $(n=63)$ once after their operation upon awakening in the ICU. Delirium was diagnosed using the confusion assessment method for the intensive care unit (CAM-ICU). The incidence of post-operative delirium in the risperidone group was lower than the control group $(11.1 \%$ versus $31.7 \%, p=.017)$. However, the effect was only shown on the day of surgery (post-operative day 0 ), with no differences in the following days between patients in the two groups. There were no changes in the length of ICU stay (3.3 days in risperidone group versus 3.2 days in placebo group) or length of hospital stay (10.5 days in risperidone group versus 10.3 days in placebo group). Hakim et al. ${ }^{(15)}$ published a randomized, double-blind, placebo-controlled, parallel-arm study, where 101 consecutive elderly patients who experienced subsyndromal delirium (SSD) after onpump cardiac surgery were enrolled between December 2007 and November 2010 at the Cardiothoracic Surgery Unity in Ain Shams University Hospital, Egypt. SSD was identified if patients had some, but not all, features of delirium on the Intensive Care Delirium Screening Checklist (ICDSC). Out of 243 subjects who underwent open-heart procedures with cardiopulmonary bypass, 101 were randomized to receive risperidone $(n=51)$ or placebo $(n=50)$. Patients with a Mini Mental State Examination (MMSE) score of less than 25 were excluded, as well as patients with hearing or visual impairment, Parkinson's disease, and a previous history of cerebrovascular disease. The results showed that risperidone $0.5 \mathrm{mg}$ twice daily by mouth given to patients after on-pump cardiac surgery (CABG, valve) could reduce the progression of subsyndromal delirium (SSD) to delirium in patients older than 65 years old. ${ }^{(15)}$ Among the patients who experienced SSD post-operatively, the risperidone group had less delirium compared to the placebo group $(13.7 \%$ versus $34 \%$ respectively, $p=.031)$. There was no effect on the duration of delirium, or the ICU or hospital lengths of stay.

\section{Olanzapine}

Larsen et al. ${ }^{(17)}$ published the only study with olanzapine, which was a double-blind randomized controlled trial with patients undergoing elective hip or knee replacements at the England Baptist Hospital in Boston, MA, from 2005 to 2007. The patients screened for the trial included all patients age 65 years or older and those less than 65 years old with a history of post-operative delirium who were scheduled for elective total knee- or total hip-replacement surgery. Patients with dementia were excluded. Four hundred and ninety-five patients were stratified and 95 patients dropped out before receiving the study drug. Therefore, 400 patients completed the study (intervention group $n=196$, placebo group $n=204$ ). The mean age was similar in both groups (73.4 years in olanzapine group and 74.0 in placebo group). The intervention group was given a total $10 \mathrm{mg}$ of olanzapine by mouth in two divided doses: $5 \mathrm{mg}$ immediately before the surgery and $5 \mathrm{mg}$ immediately after the surgery. Delirium was diagnosed using the DSM-III-R criteria. There was a reduced incidence of post-operative delirium in the olanzapine group compared to placebo $(14.3 \%$ versus $39.6 \%, p<.0001)$. However, the patients receiving olanzapine who developed delirium had a more severe and prolonged delirium that was attributed to the greater anticholinergic property of this antipsychotic $(2.2$ days versus 1.6 days, $p=.02$ ).

\section{Systematic Reviews with Meta-analyses}

Three systematic reviews with meta-analyses were published in 2013 regarding antipsychotic prophylaxis for POD. ${ }^{(9,10,11)}$ Gilmore and Wolfe ${ }^{(10)}$ analyzed five randomized clinical trials, four of which were double-blinded, as detailed above. ${ }^{(7,13,14,16,17)}$ There was a positive effect of prophylactic antipsychotic to prevent POD (14.4\% in intervention group versus $27.1 \%$ in placebo group) with a risk reduction of $12.7 \%$. Based on this risk reduction, eight patients would need to be given antipsychotic prophylaxis to prevent one case of delirium. Teslyar et al. ${ }^{(9)}$ analyzed the same five trials and showed that prophylaxis with antipsychotics reduces the incidence of delirium, with a number needed to treat (NNT) from 4.00 to 12.6 , and a relative risk ratio of 0.51 . Hirota and $\mathrm{Kishi}^{(11)}$ analyzed six randomized clinical trials, ${ }^{(7,13,14,15,16,17)}$ where the study by Hakim et al. ${ }^{(15)}$ regarding subsyndromal delirium was added. The data showed that antipsychotics significantly reduce the incidence of POD compared to placebo with a relative risk ratio of 0.50 and a NNT of 7 . There was no significant change in the length of hospital stay, duration of delirium, or severity of delirium with prophylactic antipsychotics compared to placebo in two reviews with meta-analyses. ${ }^{(10,11)}$

\section{Cholinesterase Inhibitors}

The central cholinergic system is strongly implicated in the neuropathogenesis of delirium. Specifically, under a variety of conditions such as brain hypoxia, cerebrovascular accident, infections, or trauma, there is a reduced synthesis and release of central acetylcholine that lead clinically to confusion and delirium. ${ }^{(8)}$ Also, the prevalence of delirium is higher in patients with an increased serum anticholinergic activity level. ${ }^{(18)}$ These observations lead to clinical trials where cholinesterase inhibitors were administered pre-operatively to prevent delirium. Five double-blind randomized controlled 
trials of cholinesterase inhibitors have been published; three with donepezil and two with rivastigmine. ${ }^{(19,20,21,22,23)}$ The total number of patients enrolled was 270 . None of these studies found a significant reduction in the incidence of POD compared to placebo.

\section{Donepezil}

Lipztin et al. ${ }^{(21)}$ published a pilot trial where they recruited patients over the age of 50 who were scheduled for elective total knee or hip arthroplasty between May 2000 and April 2003 at Baystate Medical Center in Springfield, Massachusetts. Patients who were currently using donepezil, with gastroesophageal reflux disase, or sick-sinus syndrome were excluded. Ninety patients were randomized to donepezil or placebo, although 10 patients were not operated on or took no study medication. Therefore, 80 patients truly completed the study (intervention group $n=39$, placebo $n=41$ ). The intervention group was administered $5 \mathrm{mg}$ of donepezil daily for one month (two weeks before and two weeks after the surgery). Delirium was diagnosed using the DSM-IV criteria. The mean age was 67 years old and the average pre-operative Mini-Mental State exam score was 29/30 in both groups. There was no change in the incidence of post-operative delirium (21\% in intervention group, versus $17 \%$ control group, $p=.69$ ). There was also no change in the mean duration of post-operative delirium (1.0 days in intervention group versus 1.3 days in placebo group, $p=.12$ ). Sampson et al. ${ }^{(19)}$ gave $5 \mathrm{mg}$ of donepezil daily for four consecutive post-operative days to patients with elective hip joint replacement. This was a double-blind, placebo controlled, parallel group randomized feasibility study conducted at the Royal Free Hospital NHS Trust (London, UK) between October 2003 and January 2004. Patients with a MMSE score of less than 26 were excluded. The diagnosis of delirium was made using the DSI scale. Fifty patients were randomized, but 14 withdrew after randomization. Thirty-three patients completed the trial (donepezil group $n=19$, placebo group $n$ $=14$ ). The mean age was 68 years old. The overall incidence of post-operative delirium was $21.2 \%$. There was a decrease of post-operative delirium in the intervention group compared to placebo but it was not statistically significant $(9.5 \%$ versus $35.7 \%$, respectively, $p=.08$ ). There was no change in the mean length of hospital stay (9.9 days in donepezil group versus 12.1 days in placebo group, $p=.09)$. Marcantoni et al. ${ }^{(20)}$ were the only authors who studied a cholinesterase inhibitor in an acute setting, with 16 patients aged 70 and older who had hip fractures. Patients admitted to the orthopedics service of a large academic medical centre were screened from January 2007 to August 2008. Those currently on cholinesterase inhibitor with advanced dementia or pathologic fracture due to metastatic cancer were excluded. Sixty patients were approached for participation, and 16 patients provided informed consent and were enrolled in the study (intervention group $n=7$, placebo $n=9$ ). The mean age was 87 years old. Donepezil 5 $\mathrm{mg}$ daily was given for 30 days. There was no change in the incidence of post-operative delirium over a six-week period (64\% versus 64\%) using the CAM criteria and the Memorial Delirium Assessment Scale (MDAS).

\section{Rivastigmine}

Similar conclusions were drawn from the two rivastigmine trials. ${ }^{(22,23)}$ Gamberini et al. ${ }^{(23)}$ published a double-blind, randomized, placebo-controlled trial where patients 65 years or older undergoing elective cardiac surgery (CABG and/or valve repair) were enrolled from February 2006 until July 2007 at the Division of Cardiac Surgery at the University Hospital Basel, Basel, Switzerland. Patients with previous or ongoing treatment with cholinesterase inhibitors were excluded, as well as patients with a MMSE score of less than 15 . The CAM was used to diagnose delirium. One hundred and twenty patients were randomized and 113 patients completed the study (intervention group $n=56$, placebo group $n=57$ ). The intervention group was given $1.5 \mathrm{mg}$ of rivastigmine by mouth three times a day for seven days. The mean age was 74 years old. The overall incidence of post-operative delirium was $31.0 \%$. There was no significant difference of post-operative delirium between the intervention and the control groups ( $32.1 \%$ versus $29.8 \%$, respectively, $p=.8)$. There was also no change in the median duration of delirium in both groups ( 3 days in placebo group and 2.5 days in rivastigmine group, $p=.3$ ) and length of hospital stay (both groups with median of 13 days). A transdermal patch of rivastigmine was used instead by Zaslavsky et al. ${ }^{(22)}$ to bypass the enteral malabsorption encountered after a surgery. It was a randomized, placebo-controlled, double-blind clinical trial where patients of 65 years and older admitted for elective surgery under general anesthesia were screened. Only the patients at risk for developing post-operative delirium were eligible (MMSE score of less than 24, history of prior delirium, severe illness, pre-operative use of psychoactive drugs, and advanced age $>$ 70 years). The $5 \mathrm{~cm}^{2}$ patch was applied for 24 hours pre-operatively in patients of the intervention group. Twenty-eight patients completed the study (11 rivastigmine and 17 placebo). The overall incidence of post-operative delirium was $21.4 \%$. There was a reduced incidence of post-operative delirium in the intervention group but it was not statistically significant $(18 \%$ versus $23 \%, p=.785)$. This study was halted prematurely because of a warning by the rivastigmine manufactuer (Novartis) about the possible increased risk of mortality in critically ill patients receiving oral rivastigmine. ${ }^{(24)}$

\section{Statins}

It has been hypothesized in animal and human models that statins have neuroprotective effects through anti-inflammatory and immunomodulatory properties in strokes, multiple sclerosis, or Alzheimer's disease. ${ }^{(25,26)}$ Three observational studies were published where authors tried to find a similar neuroprotective effect of statins against POD..$^{(27,28,29)}$ The 
conclusions differed from one to another. Redeilmeier et al. ${ }^{(29)}$ published a retrospective cohort study with more than 280,000 patients who had elective surgery. The incidence of delirium was significantly increased in the group of patients receiving statins in the year before the surgery compared to the group who did not take statins $(1.4 \%$ versus $1.1 \%, p<.001)$. This association was not observed with other cardiovascular medications such as antihypertensives or antiplatelet therapy. Katznelson et al. ${ }^{(28)}$ found instead a reduction of post-operative delirium in a prospective observational study in patients with cardiac surgery. The group that was taking statins had less delirium than the other group (10.8\% versus $12.8 \%)$. Finally, the observational cohort study of Mariscalco et al. ${ }^{(27)}$ in patients undergoing coronary artery bypass graft found no reduction of POD in the group taking statins prior to surgery $(10.8 \%$ versus $12.8 \%$, respectively, $p=.18)$.

\section{Corticosteroids}

Delirium is also partially explained by a neuroinflammatory process where proinflammatory cytokines cause central synaptic dysfunction and neuronal damage. ${ }^{(8)}$ This inflammatory state, which can be triggered by cardiac procedures on cardiopulmonary bypass for example, may respond to potent anti-inflammatory drugs such as corticosteroids. ${ }^{(30)}$ Mardani and Bigdelian ${ }^{(31)}$ published one study where dexamethasone was used to prevent POD. The study was carried out in Chamran Heart Center, a teaching hospital of Isfahan University, Iran. All male and female patients undergone elective CABG on cardiopulmonary bypass were screened and included in the study from January 2009 to February 2011. It was a randomized controlled trial with 93 patients (dexamethasone group $n=43$, placebo group $n=50$ ). Patients older than 80 years of age or with a history of delirium were excluded. Delirium was diagnosed with the DSM-IV criteria. The mean ages were 65 years old in the dexamethasone group and 60 years old in the placebo group. The subjects were given $8 \mathrm{mg}$ of dexamethasone intravenously before and after the surgery. The dexamethasone group had less post-operative delirium compared to placebo ( $16.3 \%$ versus $38 \%$, respectively, $p=.03$ ), as well as a decreased ICU length of stay ( 2.86 days versus 3.68 days, $p=.004)$ and hospital length of stay (12.93 days versus 13.64 days, $p=.02$ ). However, the reduction of POD was only statistically significant on the first post-operative day $(9.3 \%$ in dexamethasone group versus $26 \%$ in placebo group, $p=.03$ ) and not the following days (4.7\% versus $10 \%$, $p=.40$ on post-operative day $2 ; 2.3 \%$ versus $2 \%, p=1$ on post-operative day 3 ). There were more hyperglycemias in the dexamethasone group, but no significant differences on post-operative infections compared to placebo group.

\section{Gabapentin}

Studies have shown that pre-operative and post-operative pain confers a greater risk to develop POD in the elderly. ${ }^{(32,33,34)}$
Gabapentin, an anticonvulsant often used for neuropathic pain, appears to also decrease the level of post-operative pain. ${ }^{(35)}$ Leung et al. ${ }^{(36)}$ published a pilot clinical trial using gabapentin to control post-operative pain and hence prevent POD. This study was conducted in 2005 at the University of California, San Francisco Medical Center. Patients who were 45 years of age and older, undergoing surgery involving the spine, and requiring general anesthesia were included. Patients already taking pre-operative gabapentin were excluded. Twenty-one patients were enrolled (gabapentin group $n=9$, placebo group $n=12$ ). Delirium was diagnosed using the CAM. The mean ages were 57 years old in the gabapentin group and 61 years old in the placebo group. The intervention group was given gabapentin $900 \mathrm{mg}$ once daily for four consecutive days, with the first dose being given before the surgery. There was a reduction of post-operative delirium in the gabapentin group compared to placebo $(0 \%$ versus $41.7 \%$, $p=.045)$. Also, the gabapentin group had a reduced use of intravenous patient-controlled analgesia (PCA) hydromorphone in the first two post-operative days. Interestingly, no patients had an agitated delirium. One patient in each group had post-operative sedation.

\section{Benzodiazepines}

Since sleep disturbance is a core feature in delirium, Aizawa et $a l^{(37)}$ published a non-double blinded controlled clinical trial using different sedative agents to improve sleep after surgery and thus prevent POD. A total of 42 consecutive patients aged over 70 years old, but less than 86 years old, who underwent gastric or colorectal cancer resections under general anesthesia were enrolled in Hiratsuka City Hospital, Japan. Patients with "mental disorders" were excluded. Delirium was diagnosed using the DSM-IV criteria. Two patients dropped out due to incomplete administration of the agents. Forty completed the study (intervention group $n=40$, placebo group $n=40$ ) with a mean age of 76 years old in both intervention and non-intervention groups. The intervention group received the "delirium-free protocol" (DFP), which is intramuscular diazepam, intravenous flunitrazepam infusion, and intravenous pethidine infusion for the first three post-operative nights, from 8:00 pm until 4:00 am. The non-DFP group did not receive a placebo. There was a significant reduction of post-operative delirium in the intervention group $(35 \%$ versus $5 \%, p=.023)$. However, eight patients in the DFP group (40\%) developed "morning lethargy".

\section{Melatonin}

The melatonin pathway has had increased attention in the delirium literature recently. ${ }^{(38)}$ Melatonin plays a significant role in the regulation of the circadian rhythm and sleep-wake cycle, the latter being a core feature in delirium. Also, it has been demonstrated that patients with hyperactive delirium have lower urinary levels of melatonin compared to non-delirious 
patients. ${ }^{(39)}$ Three double-blinded randomized controlled trials have been published: two with melatonin, and one with tryptophan. ${ }^{(40,41,42)}$ The results of a double-blind randomized controlled trial not yet published with ramelteon was also available through a conference abstract. ${ }^{(43)}$

\section{Melatonin}

The study by Sultan ${ }^{(40)}$ is the only one that found a reduction of POD with melatonin. It was a controlled randomized double-blind study where patients older than 65 years of age who underwent elective hip arthroplasty under spinal anesthesia were included. Patients with dementia, intracranial events (e.g., stroke, bleeding, infection) and psychoactive drugs were excluded. Three hundred patients were tried to be enrolled in the study; 78 were excluded before of pre-operative delirium, 11 were further excluded due to the need to induce general anesthesia for different causes, and 8 more were excluded for post-operative ICU admission. Two hundred and three patients completed the study. The mean age was 71 years old. Delirium was diagnosed using the Abbreviated Mental Test (AMT). The patients were separated in four groups and were given placebo $(n=49)$, melatonin $(n=53)$, midazolam $(n=$ $50)$, or clonidine $(n=51)$ before the surgery. The melatonin group a significant reduction of POD compared to placebo $(0.4 \%$ versus $32.7 \%)$. Interestingly, $26 \%$ of the patients were excluded because they were in delirium before the operation. De Jonghe et al. ${ }^{(41)}$ studied the administration of melatonin in patients aged 65 years or older who were admitted for emergent acute hip fractures repair. It was a multi-centre, double-blind, randomized controlled trial between November 2008 and May 2012 in one academic and two non-academic hospitals in The Netherlands. Patients already in delirium were excluded. Four hundred and fifty-two patients were randomized, and 74 patients were excluded. The trial involved 378 patients (melatonin group $n=186$, placebo $n=192$ ) who were given $3 \mathrm{mg}$ of melatonin on the evening on admission for a total of five days. The mean age was 84 years old. No reduction of POD was noted $(25.5 \%$ in control group versus $29.6 \%$ in intervention group, $p=.4$ ), nor the duration of delirium, its severity, or the functional outcome of the patients at three months using Katz-ADL score.

\section{Tryptophan}

Tryptophan, which is an essential amino acid synthesised to serotonin and then melatonin, has also been studied. Low-levels of tryptophan serum activity have been measured post-operatively in patients, which lead to the trials by Robinson et al. in 2008 and 2014. ${ }^{(43,44)}$ The 2014 study was a randomized, double-bind, placebo-controlled trial with individuals aged 60 and older undergoing major surgery (abdominal, non-cardiac thoracic, cardiac, vascular) with an ICU stay at the Denver Veterans Affairs Medical Center, Colorado, between December 2008 and December 2012. Patients who underwent an emergency surgery were excluded. Three hundred and twenty-five patients were enrolled, and 24 patients were withdrawn from the study; 301 patients completed the study (intervention group $n=152$, placebo $n=149$ ). The mean age was 69. Delirium was diagnosed using the CAM-ICU criteria. Tryptophan $1 \mathrm{~g}$ was administered orally three times a day after the surgery for a total of nine days, or until ICU discharge. The overall incidence of post-operative delirium was $39 \%$. Even though the tryptophan serum level was significantly higher in the intervention group post-operatively, there was no reduction of the incidence of delirium ( $40 \%$ in intervention group versus $37 \%$ in control group, $p=.60$ ), and no reduction in the duration of delirium (2.9 days in intervention group versus 2.4 days in placebo group, $p=.12$ )

\section{Ramelteon}

Yamaguchi et al. ${ }^{(43)}$ completed a double-blinded randomized controlled trial using ramelteon. It is a sleep agent that binds specifically to MT1 and MT2 receptors in the hypothalamus. They enrolled 42 patients who were 70 years old or older who underwent elective total knee replacement. The intervention group was given ramelteon $8 \mathrm{mg}$ orally every night for four days. There was no statistically significant difference of POD between the two groups ( $0 \%$ in ramelteon group versus $9.2 \%$ in placebo, $p=0.48$ ) or subsyndromal delirium. Only the abstract was available at the time of this review.

A summary of all clinical studies reviewed is found in Table 2 .

\section{DISCUSSION}

Delirium is a very complex confusional syndrome the pathophysiology of which remains to be fully understood. Many mechanisms, likely intertwined, play a role. Studies suggest that an imbalance of neurotransmitters such as acetylcholine deficiency and dopamine excess may be responsible for delirium. ${ }^{(8)}$ This is what led to the use of either cholinesterase inhibitors or antipsychotics to prevent POD. Surgery creates a proinflammatory cascade causing brain edema and hypoperfusion. It increases the risk of gaseous, fat, or lipid cerebral microemboli, causing acute and prolonged cognitive dysfunction. ${ }^{(45)}$ Medications with anti-inflammatory or anti-thrombotic properties, such as dexamethasone and statins, have therefore been studied, hoping they could prevent POD. ${ }^{(27,28,29,31)}$ Since the disruption of the sleep-wake cycle is a core feature in delirium, the idea that disturbances of the metabolism of melatonin and its derivatives led to delirium is interesting. ${ }^{(38)}$ Objective measurements have been made, but the causal relation is still unclear. ${ }^{(38,39,44)}$

Each of these studies targeted one putative mechanism of delirium, and the results are therefore limited. The few studies that found a reduction of the incidence of POD were mostly studies with a small population or non-double blind 


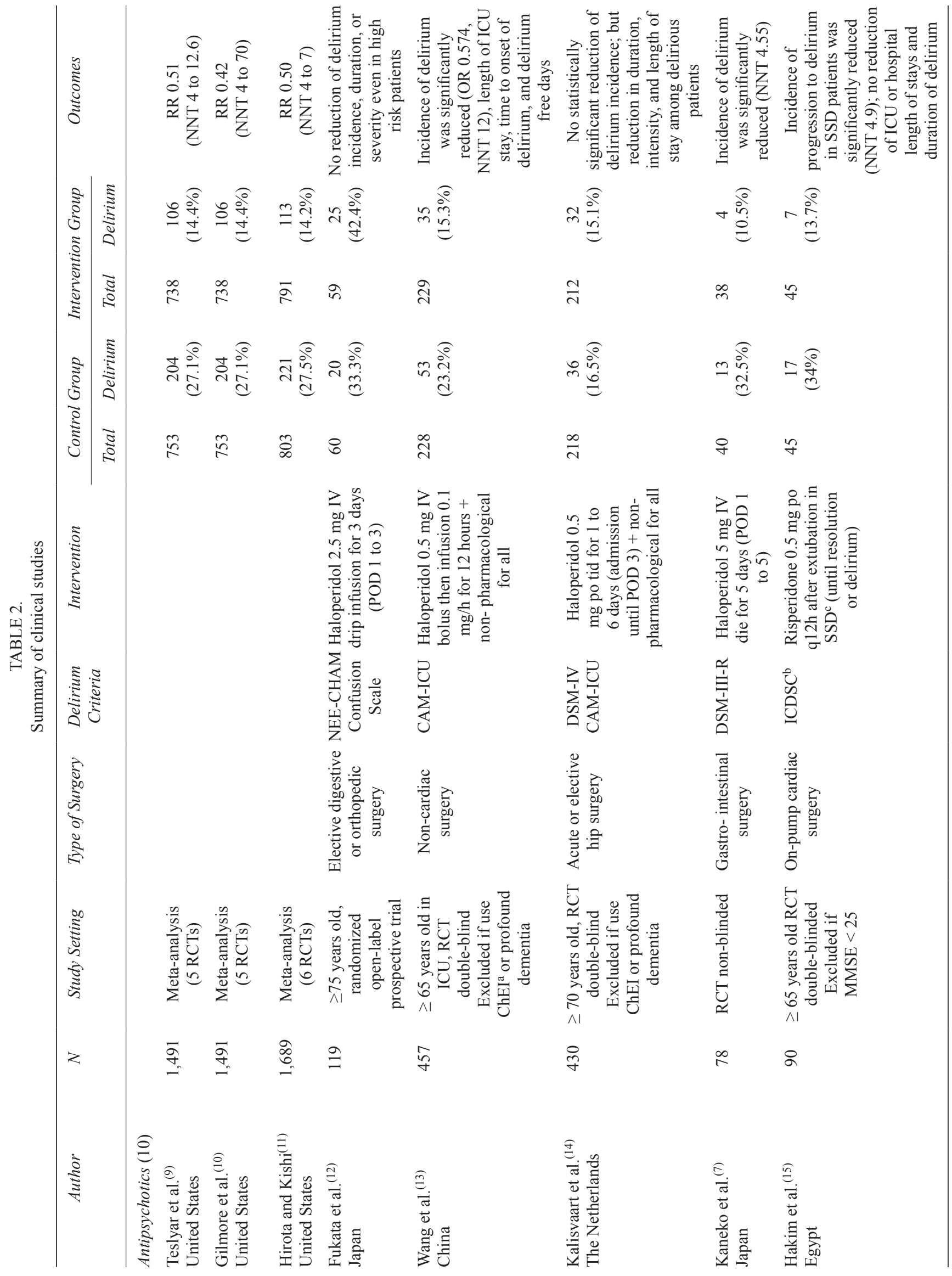




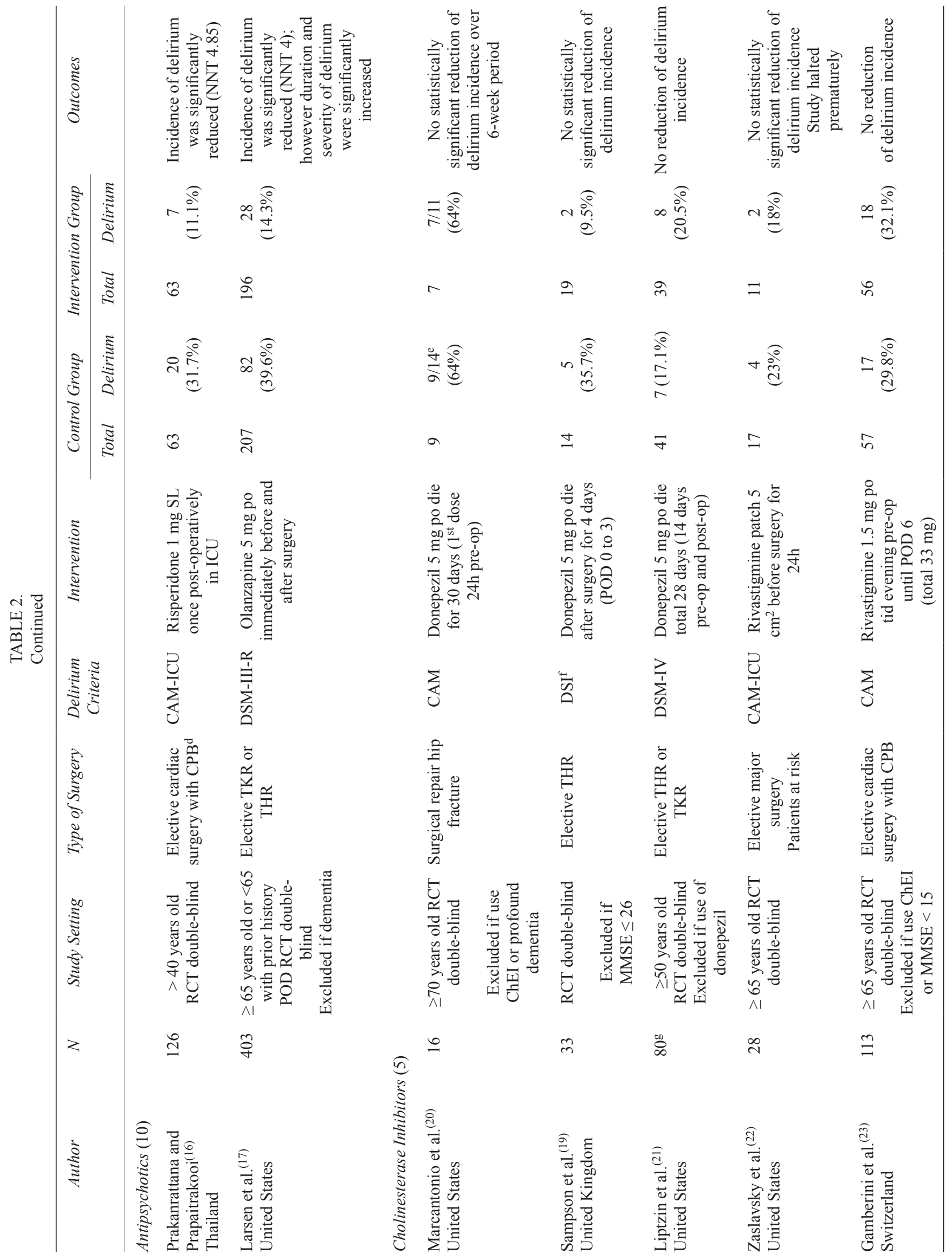




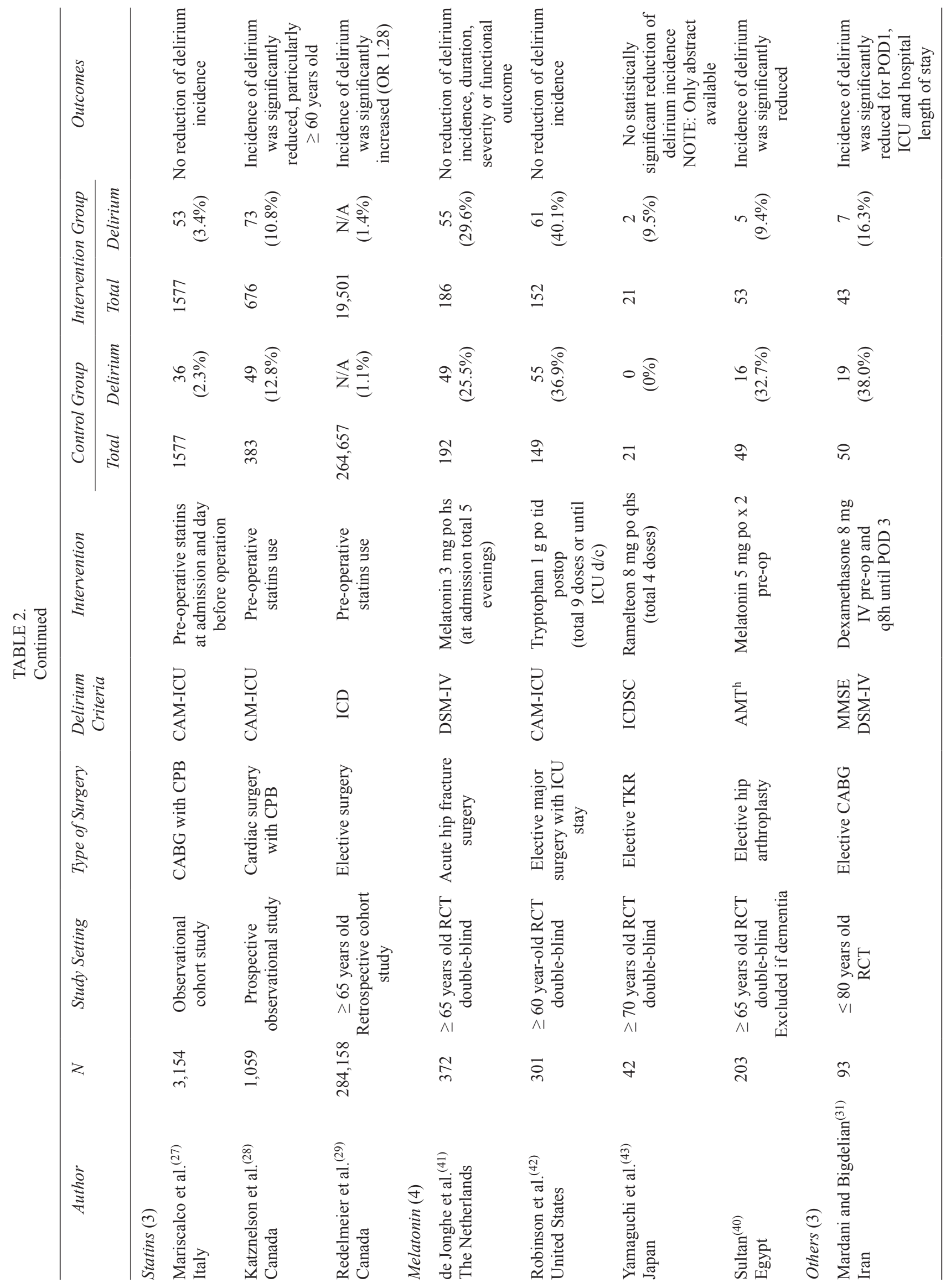


TREMBLAY: POST-OP PREVENTION OF DELIRIUM

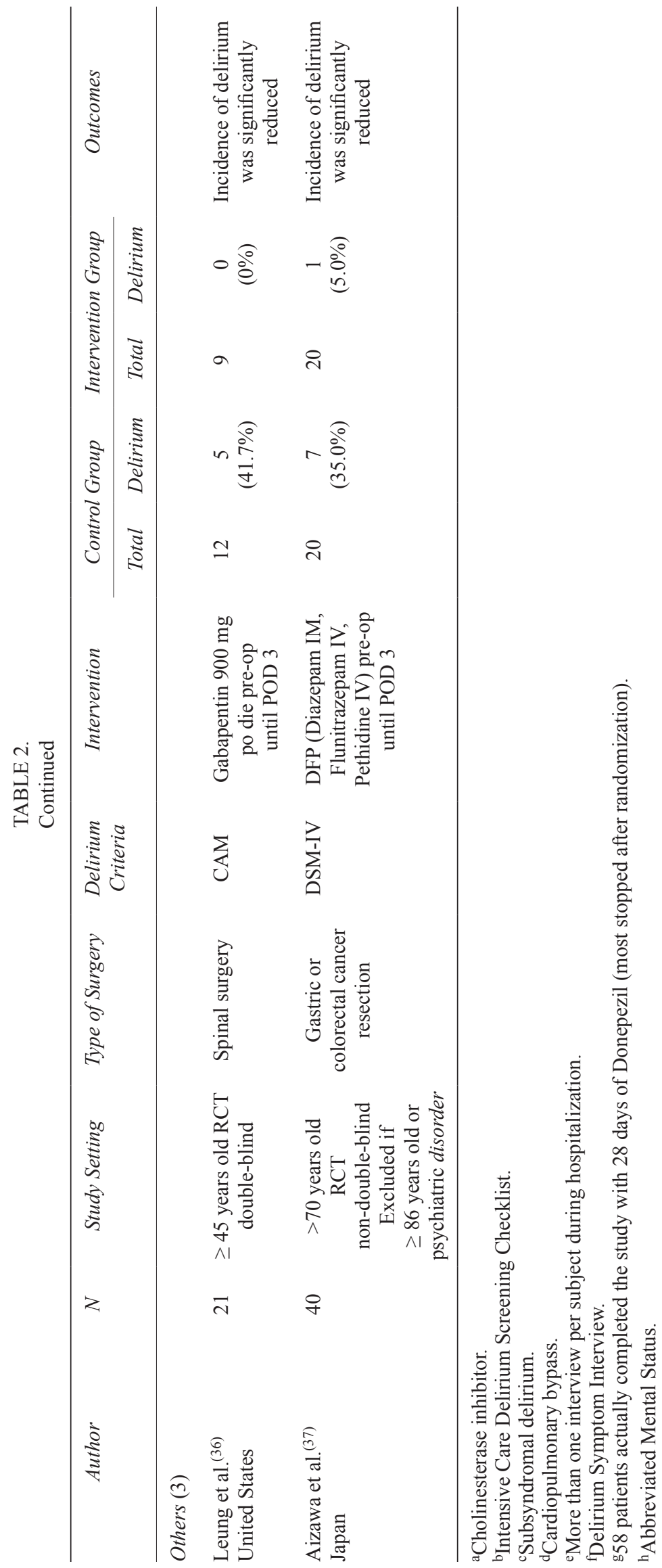


randomized controlled trials. . $^{(7,13,15,16,17,28,31,36,37,40)}$ The study by Wang et al. ${ }^{(13)}$ was the biggest well-designed clinical trial, with 457 subjects, that found a reduction of POD with haloperidol. However, its route of administration (by infusion drip) is probably not suited for a non-research setting and requires constant monitoring by nurses. Also, in many of these studies, patients at higher risk of delirium are excluded, specifically very elderly subjects, ${ }^{(31,37)}$ patients with established cognitive impairment, ${ }^{(15,17,19,20,23,37,40)}$ and those with polypharmacy with psychoactive drugs. ${ }^{(13,17,23,40,42)}$ Moreover, a significant proportion of the trials enrolled a younger population in its sixth decade of life. ${ }^{(16,19,21,31,36,42)}$ The studies of Leung et $a l .{ }^{(36)}$ with gabapentin and Aizawa et al. ${ }^{(37)}$ with the DFP protocol, where there was reduction of POD, raises a question of whether a hypoactive state of delirium could have been precipitated instead by these sedative medications, and therefore misdiagnosed as "non-delirious". It is well known that benzodiazepines are a major risk factor for non-alcoholic delirium. ${ }^{(46)}$ Also, intramuscular injections and intravenous infusion post-operatively may not be the best patient care or easily feasible by the nursing staff. Regarding statins, it is difficult to interpret the causal relation between statins and post-operative delirium since none of these studies were randomized controlled trial but observational studies. ${ }^{(27,28,29)}$ Only one study found a significant benefit using melatonin compared to placebo to prevent POD. ${ }^{(40)}$ However, patients at higher risk of POD who would most benefit from pre-operative prophylaxis were excluded (sensory impairment, dementia, electrolytes disturbances, psychoactive medications). Also, the sedation score in the melatonin group was significantly increased compared to control group; it is unclear if these patients could have been in an hypoactive delirium, which may be associated with a poorer prognosis than the hyperactive subtype since it often goes unrecognized and therefore is not treated promptly. ${ }^{(4)}$ The dexamethasone study showed a significant reduction of POD compared to placebo, but the effect was only significant on the first post-operative day. ${ }^{(31)}$ Cholinesterase inhibitor prophylaxis has no positive effect on POD. ${ }^{(20,21,22,23,24)}$

Delirium is not only an acute medical complication, its consequences can persist for months. An ideal medication would be one that could alter this "natural course", such as by decreasing the hospital length of stay, increasing the rate of discharge to a rehabilitation center, decreasing institutionalization, or protecting the brain from further cognitive impairment. Until more studies show promising results, the well-described non-pharmacological measures to prevent delirium will continue to be the gold standard.

\section{CONCLUSION}

Post-operative delirium is a frequent and serious surgical complication in the elderly population. The short-term and long-term consequences can be disastrous, and include death or severe functional impairment with institutionalization.
Non-pharmacological measures and comprehensive geriatric care reduce the incidence or severity of post-operative delirium. However, the current data on pharmacological agents are much more controversial and debatable. The strongest evidence, still very limited, is for haloperidol, but the appropriate dose and route of administration remain to be determined. The risk and benefits need to be considered, especially with recent studies that found an increased risk of mortality in patients with dementia taking antipsychotics. ${ }^{(48)}$ Until further studies are published, non-pharmacological measures should be the mainstay of treatment to reduce and prevent post-operative delirium in the elderly.

\section{CONFLICT OF INTEREST DISCLOSURES}

The authors declare that no conflicts of interest exist.

\section{REFERENCES}

1. Inouye SK, van Dyck CH, Alessi CA, et al. Clarifying confusion: the confusion assessment method. A new method for detection of delirium. Ann Intern Med. 1990;113(12):941-48.

2. Dyer CB, Ashton CM, Teasdale TA. Postoperative delirium: a review of 80 primary data-collection studies. Arch Intern Med. 1995;155(5):461-65.

3. Bilotta F, Lauretta MP, Borozdina A, et al. Postoperative delirium: risk factors, diagnosis and perioperative care. Minerva Anestesiol. 2013;79(4):1066-76.

4. Girard TD, Jackson JC, Pandharipande PP, et al. Delirium as a predictor of long-term cognitive impairment in survivors of critical illness. Crit Care Med. 2010;38(7):1513-20.

5. Leslie DL, Inouye SK. The importance of delirium: economic and societal costs. J Am Geriatr Soc. 2011;59(Suppl 2):S241-S243.

6. Martinez F, Tobar C, Hill N. Preventing delirium: should non-pharmacological, multicomponent interventions be used? A systematic review and meta-analysis of the literature. Age Ageing. 2015;44(2):196-204.

7. Kaneko T, Cai J, Ishikura T, et al. Prophylactic consecutive administration of haloperidol can reduce the occurrence of postoperative delirium in gastrointestinal surgery. Yonago Acta Medica. 1999;42(3):179-84.

8. Maldonado JR. Neuropathogenesis of delirium: review of current etiologic theories and common pathways. Am J Geriatr Psychiatry. 2013;21(12):1190-222.

9. Teslyar P, Stock VM, Wilk CM, et al. Prophylaxis with antipsychotic medication reduces the risk of post-operative delirium in elderly patients: a meta-analysis. Psychosomatics. 2013;54(2):124-31.

10. Gilmore ML, Wolfe DJ. Antipsychotic prophylaxis in surgical patients modestly decreases delirium incidence - but not duration -in high-incidence samples: a meta-analysis. Gen Hosp Psychiat. 2013;35(4):370-75.

11. Hirota T, Kishi T. Prophylactic antipsychotic use for postoperative delirium: a systematic review and meta-analysis. J Clin Psychiat. 2013;74(12):e1136-44. 
12. Fukata S, Kawabata Y, Fujisiro K, et al. Haloperidol prophylaxis does not prevent postoperative delirium in elderly patients: a randomized, open-label prospective trial. Surg Today. 2014;44(12):2305-13.

13. Wang W, Li HL, Wang DX, et al. Haloperidol prophylaxis decreases delirium incidence in elderly patients after noncardiac surgery: a randomized controlled trial. Crit Care Med. 2012;40(3):731-39.

14. Kalisvaart KJ, de Jonghe JF, Bogaards MJ, et al. Haloperidol prophylaxis for elderly hip-surgery patients at risk for delirium: a randomized placebo-controlled study. J Am Geriatr Soc. 2005;53(10):1658-66.

15. Hakim SM, Othman AI, Naoum DO. Early treatment with risperidone for subsyndromal delirium after on-pump cardiac surgery in the elderly: a randomized trial. Anesthesiology. 2012;116(5):987-97.

16. Prakanrattana U, Prapaitrakool S. Efficacy of risperidone for prevention of postoperative delirium in cardiac surgery. Anaesth Intensive Care. 2007;35(5):714-19.

17. Larsen KA, Kelly SE, Stern TA, et al. Administration of olanzapine to prevent postoperative delirium in elderly joint-replacement patients: a randomized, controlled trial. Psychosomatics. 2010;51(5):409-18.

18. Tune LE. Serum anticholinergic activity levels and delirium in the elderly. Semin Clin Neuropsychiat. 2000;5(2):149-53.

19. Sampson EL, Raven PR, Ndhlovu PN, et al. A randomized, double-blind, placebo-controlled trial of donepezil hydrochloride (Aricept) for reducing the incidence of postoperative delirium after elective total hip replacement. Int J Geriatr Psychiat. 2007;22(4):343-49.

20. Marcantonio ER, Palihnich K, Appleton P, et al. Pilot randomized trial of donepezil hydrochloride for delirium after hip fracture. J Am Geriatr Soc. 2011;59(Suppl 2):S282-S288.

21. Liptzin B, Laki A, Garb JL, et al. Donepezil in the prevention and treatment of post-surgical delirium. Am J Geriatr Psychiat. 2005;13(12):1100-06.

22. Zaslavsky A, Haile M, Kline R, et al. Rivastigmine in the treatment of postoperative delirium: a pilot clinical trial. Int $J$ Geriatr Psychiat. 2012;27(9):986-88.

23. Gamberini M, Bolliger D, Lurati Buse GA, et al. Rivastigmine for the prevention of postoperative delirium in elderly patients undergoing elective cardiac surgery--a randomized controlled trial. Crit Care Med. 2009;37(5):1762-68.

24. van Eijk MM, Roes KC, Honing ML, et al. Effect of rivastigmine as an adjunct to usual care with haloperidol on duration of delirium and mortality in critically ill patients: a multicentre, double-blind, placebo-controlled randomised trial. Lancet. 20101376(9755):1829-37.

25. Stepień K, Tomaszewski M, Czuczwar SJ. Neuroprotective properties of statins. Pharmacol Rep. 2005;57(5):561-69.

26. Blanco-Colio LM, Tunón J, Martín-Ventura JL, et al. Antiinflammatory and immunomodulatory effects of statins. Kidney Int. 2003;63(1):12-23.

27. Mariscalco G, Cottini M, Zanobini M, et al. Preoperative statin therapy is not associated with a decrease in the incidence of delirium after cardiac operations. Ann Thorac Surg. 2012;93(5):1439-47.
28. Katznelson R, Djaiani GN, Borger MA, et al. Preoperative use of statins is associated with reduced early delirium rates after cardiac surgery. Anesthesiology. 2009;110(1):67-73.

29. Redelmeier DA, Thiruchelvam D, Daneman N. Delirium after elective surgery among elderly patients taking statins. CMAJ. 2008;179(7):645-52.

30. Kozora E1, Kongs S, Collins JF, et al. Cognitive outcomes after on- versus off-pump coronary artery bypass surgery. Ann Thorac Surg. 2010;90(4):1134-41.

31. Mardani D, Bigdelian H. Prophylaxis of dexamethasone protects patients from further post-operative delirium after cardiac surgery: a randomized trial. J Res Med Sci. 2013;18(2):137-43.

32. Lynch EP, Lazor MA, Gellis JE, et al. The impact of postoperative pain on the development of postoperative delirium. Anesth Analg. 1998;86(4):781-85.

33. Kosar CM, Tabloski PA, Travison TG, et al. Effect of preoperative pain and depressive symptoms on the risk of postoperative delirium: a prospective cohort study. Lancet Psychiat. 2014;1(6):431-36.

34. Vaurio LE, Sands LP, Wang Y, et al. Postoperative delirium: the importance of pain and pain management. Anesth Analg. 2006;102(4):1267-73.

35. Dirks J, Fredensborg BB, Christensen D, et al. A randomized study of the effects of single-dose gabapentin versus placebo on postoperative pain and morphine consumption after mastectomy. Anesthesiology. 2002;97(3):560-64.

36. Leung JM, Sands LP, Rico M, et al. Pilot clinical trial of gabapentin to decrease postoperative delirium in older patients. Neurology. 2006;67(7):1251-53.

37. Aizawa K, Kanai T, Saikawa Y, et al. A novel approach to the prevention of postoperative delirium in the elderly after gastrointestinal surgery. Surg Today. 2002;32(4):310-14.

38. de Rooij SE, van Munster BC. Melatonin deficiency hypothesis in delirium: a synthesis of current evidence. Rejuvenation Res. 2013;16(4):273-78

39. Balan S, Leibovitz A, Zila SO, et al. The relation between the clinical subtypes of delirium and the urinary level of 6-SMT. $J$ Neuropsychiatry Clin Neurosci. 2003;15(3):363-66.

40. Sultan SS. Assessment of role of perioperative melatonin in prevention and treatment of postoperative delirium after hip arthroplasty under spinal anesthesia in the elderly. Saudi $J$ Anaesth. 2010;4(3):169-73.

41. de Jonghe A, van Munster BC, Goslings JC, et al. Effect of melatonin on incidence of delirium among patients with hip fracture: a multicentre, double-blind randomized controlled trial. CMAJ. 2014;186(14):E547-56.

42. Robinson TN, Dunn CL, Adams JC, et al. Tryptophan supplementation and postoperative delirium--a randomized controlled trial. J Am Geriatr Soc. 2014;62(9):1764-71.

43. Yamaguchi Y, Mihara T, Taguri M, et al. Melatonin receptor agonist for the prevention of postoperative delirium in elderly patients: a randomized, double-blind, placebo-controlled trial [abstract]. Intensive Care Med. 2014;40(Suppl 1).

44. Robinson TN, Raeburn CD, Angles EM, et al. Low tryptophan levels are associated with postoperative delirium in the elderly. Am J Surg. 2008;196(5):670-74. 
45. Hála M. Pathophysiology of postoperative delirium: systemic inflammation as a response to surgical trauma causes diffuse microcirculatory impairment. Med Hypotheses. 2007;68(1):194-96.

46. Hofmann W. Benzodiazepines in der geriatrie [in German]. Z Gerontol Geriatr. 2013;46(8):769-76.

47. Peterson JF, Truman BL, Shintani A, et al. The prevalence of hypoactive, hyperactive, and mixed-type delirium in medical ICU patients [abstract]. J Am Geriatr Soc. 2003;51(Suppl 4):S174.
48. Maust DT, Kim HM, Seyfried LS, et al. Antipsychotics, other psychotropics, and the risk of death in patients with dementia: number needed to harm. JAMA Psychiat. 2015;72(5):438-45.

Correspondence to: Patrice Tremblay, MD, CCFP (COE), Department of Family Medicine, St. Mary's Hospital Center, 3830 Lacombe Avenue, Montreal, Quebec, H3T 1M5 Canada E-mail: patrice.tremblay@mcgill.ca 